\title{
Diagnosis and Management of Osteoporosis During COVID-19: Systematic Review and Practical Guidance
}

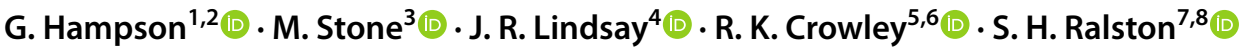

Received: 3 March 2021 / Accepted: 22 April 2021 / Published online: 18 May 2021

(c) The Author(s) 2021

\begin{abstract}
It is acknowledged that the COVID-19 pandemic has caused profound disruption to the delivery of healthcare services globally. This has affected the management of many long-term conditions including osteoporosis as resources are diverted to cover urgent care. Osteoporosis is a public health concern worldwide and treatment is required for the prevention of further bone loss, deterioration of skeletal micro-architecture, and fragility fractures. This review provides information on how the COVID-19 pandemic has impacted the diagnosis and management of osteoporosis. We also provide clinical recommendations on the adaptation of care pathways based on experience from five referral centres to ensure that patients with osteoporosis are still treated and to reduce the risk of fractures both for the individual patient and on a societal basis. We address the use of the FRAX tool for risk stratification and initiation of osteoporosis treatment and discuss the potential adaptations to treatment pathways in view of limitations on the availability of DXA. We focus on the issues surrounding initiation and maintenance of treatment for patients on parenteral therapies such as zoledronate, denosumab, teriparatide, and romosozumab during the pandemic. The design of these innovative care pathways for the management of patients with osteoporosis may also provide a platform for future improvement to osteoporosis services when routine clinical care resumes.
\end{abstract}

Keywords COVID-19 $\cdot$ Osteoporosis $\cdot$ FRAX $\cdot$ Osteoporosis management

G. Hampson

geeta.hampson@kcl.ac.uk

1 Department of Chemical Pathology and Metabolic Medicine, St Thomas' Hospital, Lambeth Palace Road, 5th Floor, North Wing, London SE1 7EH, UK

2 Department of Rheumatology, Metabolic Bone Clinic, Guy’s Hospital, London, UK

3 Metabolic Bone Service, University Hospital Llandough, Llandough, Penarth CF64 2XX, UK

4 Osteoporosis and Bone Metabolism Service, Musgrave Park Hospital, Belfast, Northern Ireland, UK

5 Department of Endocrinology, St Vincent's University Hospital, Dublin, Ireland

6 University College Dublin, Dublin, Ireland

7 Centre for Genomic and Experimental Medicine, Institute of Genetics and Cancer, University of Edinburgh, Western General Hospital, Edinburgh EH4 2XU, UK

8 Rheumatic Diseases Unit, NHS Lothian Western General Hospital Edinburgh, Edinburgh EH4 2XU, UK

\section{Introduction}

The rapid spread of infection caused by the severe acute respiratory syndrome coronavirus 2 (SARS-CoV-2) was categorised by the World Health Organisation (WHO) as a pandemic in March 2020. As of January 2021, over 90 million cases have been confirmed worldwide with over 1.9 million deaths [1]. In the UK, over 4.1 million people have tested positive with in excess of 122,000 deaths as of February 2021 [1]. In most young people SARS-CoV-2 is asymptomatic or causes mild influenza-like symptoms with loss of taste and smell [2]. However, others may develop a life-threatening illness with acute respiratory distress syndrome (ARDS) and multiorgan failure requiring hospital admission and ventilatory support [3]. Severe forms of the illness are associated with activation of the immune system, with increased production of pro-inflammatory cytokines and raised levels of CRP [4]. The risks of hospital admission and poor outcome increase markedly with age and are associated with pre-existing obesity, hypertension, cardiovascular disease, and ethnic group. Since the first reported cases of the disease in Wuhan, China in December 2019, 
a series of measures have been implemented worldwide to limit the spread of the virus including travel bans, limits on public gatherings and nationwide lockdowns. Public health approaches have included social distancing and infection control measures such as frequent hand washing, sanitisation and more recently vaccinations. Introduction of these strategies, has been partially successful in mitigating virus spread but has caused severe disruption to healthcare and social services [5]. Because of the prioritisation of urgent services and delaying of elective care, the management of many chronic or long-term medical conditions, such as osteoporosis, has been challenging as resources are diverted from chronic diseases care to combat the pandemic [6]. The potential impact of this is considerable since osteoporosis is the commonest bone disease worldwide affecting 1 in 2 women and 1 in 5 men at some point in life. It is estimated that the cost of osteoporosis is 37 billion EUR per year in the EU, and 19 billion USD per year in the USA due, in part, to hospitalisation as a result of fractures [7]. Costs are projected to rise dramatically alongside an increasing osteoporosis prevalence in coming years with a larger elderly population and it is estimated that osteoporotic fractures cause an annual global loss of 5.8 million healthy life years to disability and reduced relative survival. Hip fractures are associated with a $30 \%$ mortality rate at 1 year and $53 \%$ of patients who sustain a hip fracture are no longer able to live independently [8]. Here we have conducted a systematic review of the literature to assess the impact of the pandemic on the diagnosis and treatment of osteoporosis and documented the adaptations that have been made in osteoporosis services in five secondary referral centres in the supplementary material.

\section{Methods}

A literature review of electronic databases (PubMed, Medline, Google Scholar) was conducted by one of the authors including the following words 'COVID-19 and osteoporosis or bone or COVID-19 and fractures or fracture assessment' for the selection of studies that described the effect of the COVID-19 pandemic on fracture liaison and osteoporosis services including osteoporosis diagnosis, clinical management, and outcomes where evidence was available. The search was carried out from January 2020 to February 2021. The identification of relevant articles including review articles, practice guidelines, original articles, comment/editorial/viewpoint, letters was performed by GH. The main source of information was obtained from published articles. Criteria for inclusion were (1) written in English language, (2) publications reporting the impact of COVID-19 on bone health. The search using the terms 'COVID-19 and osteoporosis' or 'COVID-19 and bone' yielded 40 publications after duplicates were removed. Two were not written in English and a further two were published in abstract form only and were excluded. Thirty-six were reviewed and comprised of practice guidelines $(n=3)$, review articles $(n=13)$, original articles $(n=8)$, viewpoint/editorial $(n=9)$, letter $(n=3)$. Using the terms COVID-19 and fractures identified a further nine publications ( 7 original articles and 2 review articles). The selection of studies is shown in a PRISMA flow diagram (Fig. 1).

\section{Fracture Risk Assessment}

The two key steps in risk assessment of patients suspected to have osteoporosis is a fracture risk assessment which may also be coupled to a measurement of bone mineral density (BMD) $[9,10]$. There are various means of fracture risk assessment $[11,12]$ but one of the most commonly used is the FRAX calculator which is available for 66 countries covering $80 \%$ of the world population. It has been adopted in several country-specific guidelines for initiation of treatment to reduce fractures [13-15]. Although FRAX can be calculated on the basis of clinical risk factors alone and does not necessarily require attendance at hospital for a BMD measurement, a study by McCloskey and colleagues showed that the use of FRAX was significantly reduced over the 3-month period from February 2020 to April 2020 [16]. The number of sessions, defined as FRAX tool usage within a 30-min time frame, fell by $23 \%$ and 58\% in March and April 2020 respectively compared to the same period in 2019. In Europe, the majority of countries reduced their usage by at least 50\%. In Latin America, the reductions were higher than $50 \%$ with smaller reductions seen in Asian countries. The authors estimated that over the 3-month period, approximately 500,000 fewer individuals were assessed for their risk of fracture than normal [16]. The reasons for this were not explored in the study but our experience suggests that this may have been due to difficulties in patients attending their GP's and/or being referred to hospital clinics for these calculations due to diversion to resources from elective care to urgent care as the result of the pandemic.

\section{Bone Density Measurements}

Measurement of bone mineral density (BMD) by dualenergy X-ray absorptiometry (DXA) is a valuable tool for the diagnosis of osteoporosis, for improving the precision of fracture risk assessment following the use of screening tools like FRAX and for monitoring the response to osteoporosis therapy. The evidence which supports the use of osteoporosis treatments to reduce fracture risk was gained by randomised controlled trials of individuals who had low BMD values on DEXA and/or low trauma vertebral fractures [10]. Several studies have also shown that change in BMD following treatment is correlated with a reduction in fracture risk 


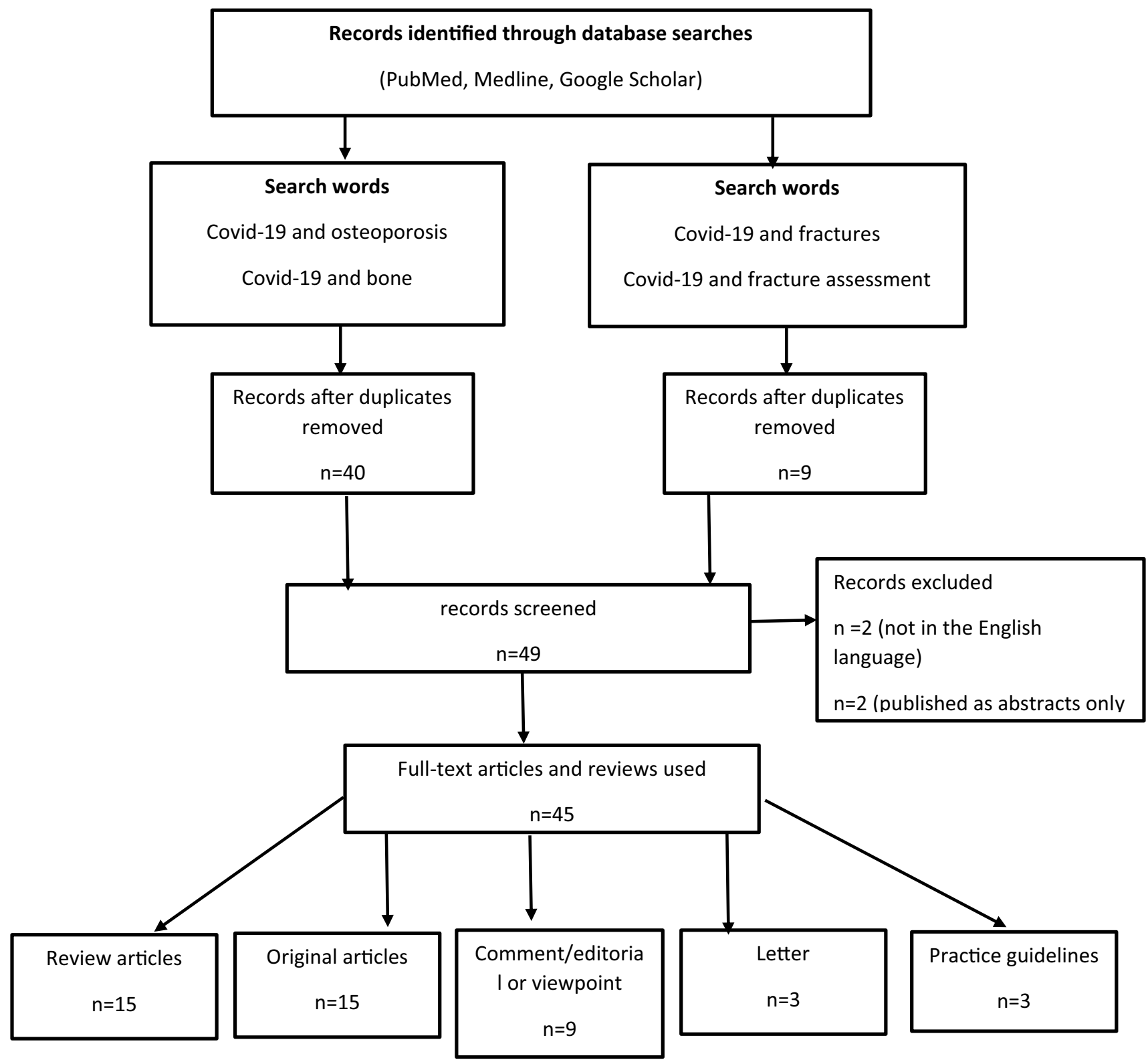

Fig. 1 PRISMA flow diagram of the search for eligible studies COVID-19 and osteoporosis or fractures

[17]. Measurements of BMD are largely provided by secondary-care facilities in the UK and this has been compromised and disrupted during the pandemic [18]. Diagnostic imaging or radiological procedures have been prioritised during the pandemic based on clinical urgency and in many centres DXA services have been deprioritised or even paused temporarily. In centres where DXA service have continued to operate the throughput has markedly diminished due to stringent infection control measures and social distancing. In addition, frail and elderly patients who are particularly susceptible to fragility fractures associated with osteoporosis have been reluctant to attend hospital for fear of contracting SARS-CoV2. Accordingly, across the UK it was estimated that there were $73 \%$ fewer DXA scans carried out in June 2020 compared to 2019 [18]. As a result of this, it is almost certain that many months may pass before the backlog is cleared and waiting lists return to acceptable levels.

\section{Biochemical Investigations}

In some centres, issues have been experienced with the availability of laboratory tests including serum calcium, creatinine, and $25(\mathrm{OH}) \mathrm{D}$ which may be measured as part of safety checks before treatment with zoledronic acid or denosumab. Biochemical markers of bone turnover such as PINP are also used in some centres to monitor adherence to 
treatment. Social distancing measures as well as the reduction in access to phlebotomy during the pandemic has meant that it has often been challenging to have these pre-treatment blood tests performed. Strategies that are being explored to surmount these issues are the establishment of "mobile" phlebotomy vans and also engagement with local pharmacies to offer phlebotomy services where GP's are finding it difficult to provide phlebotomy services.

\section{Fracture Liaison Services}

Fracture liaison services (FLS) are structured secondary prevention programmes for men and women aged 50 years or older after a fragility fracture [19]. These involve a multidisciplinary diagnostic-therapeutic pathway for the management of complications in patients with a recent fragility fracture with the aim of reducing falls and /or a new fragility fracture and ensures appropriate care and treatment in high-risk patients after discharge from hospital [20]. These patients are identified during their attendance at a fracture clinic or hospitalisation after an acute fragility fracture, often through scrutiny of the electronic patient record. Typically, those with major osteoporotic fractures aged $>50$ years are offered DXA and, depending on the results, initiation of osteoporosis treatment is recommended. Some guidelines recommend initiation of treatment in all patients with a fracture above a certain age without recourse to a DXA. The FLS have been shown worldwide to be effective clinically in reducing the risk of fractures after a first fracture [21]. In the current pandemic, many FLS including the associated rehabilitation services have largely closed as resources are diverted to the care of patients with COVID-19. A recent survey of out-patient attendances to the fracture clinic for non-hip fragility fractures in a large university hospital showed a decline during lockdown to a mean number of 26.0 (SD 7.3) from 63.1 (SD 12.6) outpatients per week prior to lockdown in 2020 and in previous years. It has been speculated that the reduction in non-hip fractures may be due in part to fewer falls as a result of movement restrictions [22]. Some centres, however, have offered virtual fracture liaison clinics during the COVID-19 pandemic leading to reduced delays in the initiation of fracture prevention therapies [23].

\section{Care of Patients with Hip Fracture}

Data on the rates of hip fracture have been conflicting during the pandemic such that some studies have reported no change compared with previous years [22], whereas others have described increases or decreases [24-26]. Delays to surgery due to limited theatre access have been reported as surgical wards have been reconfigured to cope with increasing numbers of admissions related to COVID-19 [26]. In addition, hip fracture patients may not be given adequate care following discharge through redeployment of key staff such as physiotherapists and occupational therapists to acute services. A retrospective audit highlighted a significant reduction in the review of patients with femoral neck fractures by the orthogeriatric team after the lockdown due to redeployment of staff [27]. Reductions in the prescription for calcium/vitamin D supplements and osteoporosis medications were seen at the same time which may have a significant impact on fracture burden with resultant increases in morbidity and mortality [27].

\section{Medications for Osteoporosis}

Globally, there is a documented treatment gap in the management of osteoporosis as only one-fifth of patients have been estimated to receive appropriate treatment after a hip fracture at a time where they are at highest risk of another fracture [28]. The COVID-19 pandemic has further exacerbated this gap as treatment of patients with osteoporosis is considered low on the list of clinical priorities, including zoledronic acid (ZA) which is usually delivered in hospital day units, and teriparatide and romosozumab which are typically initiated by specialists in secondary care. Denosumab can be initiated in primary care but in the UK and the ROI, treatment is usually commenced on the recommendation of an osteoporosis specialist in secondary care, although this may change in the ROI.

Capacity for delivery of infusion treatments has been reduced due to redeployment of staff to acute services, and reduced capacity for delivery of infusions due to social distancing. Finally, many patients have been unwilling or unable to attend secondary care for scheduled treatments. With ZA the timing of the next dose is not critical as its anti-resorptive effect is sustained for up to 2 years following an infusion due to its long skeletal retention time [29]. Reflecting this fact, it has been demonstrated that there is prolonged protecting against fractures after treatment with zoledronic acid [30, 31] and other oral bisphosphonates, [32, 33]. Accordingly, even if a scheduled infusion needs to be delayed for several months this is unlikely to be harmful.

Denosumab presents specific problems since its inhibitory effects on bone resorption disappear quickly when the administration is delayed beyond 7 months after the last dose $[34,35]$. Furthermore, it is now recognised that patients who stop denosumab have a rebound increase in bone remodelling for 6-12 months after stopping therapy, and this can be associated with the occurrence of multiple vertebral fractures and even hypercalcaemia [36]. Accordingly, current guidelines recommend that the delay in denosumab should not exceed 1 month from the scheduled date of injection $[35,36]$ Discontinuation of teriparatide (TPTD) also leads to bone loss over the first 12 months, but there is no evidence of a rebound increase in bone remodelling or an increased 
risk of vertebral fractures [37]. Even if TPTD needed to be stopped due to interruptions of supply, bone loss could be mitigated by prescription of an oral bisphosphonate which has been shown to maintain the increase in BMD and protect against fractures for up to 5 years $[38,39]$. Discontinuing romosozumab also leads to bone loss within 12 months and there is evidence of increased bone resorption within 3 months of stopping, although there is insufficient evidence to ascertain whether this leads to increased risk of rebound fractures [40]. If romosozumab needs to be stopped for any reason bone loss could be prevented with an antiresorptive drug such as an oral bisphosphonate.

\section{Does Osteoporosis Influence Outcome of SARS-Cov2 Infection?}

A large number of clinical risk factors have been associated with mortality in patients with COVID-19 disease, the most important of which are age, BMI, ethnic group, respiratory disease, cardiovascular disease, chronic kidney disease, chronic liver disease, neurological disease, and immunosuppression [41]. An interesting observation from the QResearch database of 1205 GP practices in England was that a history of hip, spine, humerus, and wrist fractures was associated with an increased risk of death from SARS Cov2 infection in women $(1.12,95 \%$ CI, 1.00-1.260) and in men 1.35 [1.24-1.47]. Individuals with previous fractures also had an increased risk of hospital admission due to SARS Cov2 infection [42]. Another small study investigated the prevalence of morphometric vertebral fractures (VFs) among patients with SARS Cov2 infection. This study showed that VF's were common (about 36\%) among those hospitalised with severe SARS Cov2 compared with the general population where the prevalence of VFs ranged from $18-26 \%$ in women and $8-20 \%$ in men. The presence of VFs in this study was a strong prognostic marker and predictor of clinical outcomes and disease severity as other well-described risk factors and co-morbidities, although the prevalence of VFs in the study population was higher than previously reported in the European general populations $[43,44]$. Furthermore, patients with VFs were more likely to require non-invasive mechanical ventilation $(48.8 \%$ vs $27.4 \%, p=0.02$ ). Mortality was higher in those with severe VFs (60\%) compared with those with moderate or mild VFs (7\% and 24\%, respectively) [43]. The author speculated that impaired respiratory function and kyphosis associated with VF's may decrease vital lung capacity and increase the risk of severe SARS Cov2 infection. In a recent meta-analysis the prevalence of SARS Cov2 infection in hip fracture patients ranged from 1 to $28 \%$, with a mean of $13 \%$ [45]. Data from 21 studies which reported mortality following hip fracture showed that COVID-19 positive patients have a seven-fold increased risk of death compared to COVID-19 negative patients. Crude mortality rate was $35 \%$ in those with COVID-19 infection compared to $8 \%$ in those without. However, the studies did not adjust for confounders such as age, sex, co-morbidities, level of independence, frailty which are known to be associated with mortality risk after a hip fracture. [45].

\section{Does SARS-Cov 2 Infection Predispose to Osteoporosis?}

Since COVID-19 infection leads to increased pro-inflammatory cytokine production and can be associated with prolonged immobilisation in seriously ill patients, this might be expected to increase bone resorption and promote bone loss [4].

In addition, the medium and long-term sequelae of the infection may be expected to have a negative impact on the skeleton. Evidence shows patients who have suffered severe SARS Cov2 continue to experience health problems including breathing difficulties, cardiovascular problems, loss of muscle mass, muscular weakness, mobility issues, and impaired activities of daily living [46]. Thus, people suffering from the medium to long-term effects of COVID-19 infection will need a comprehensive recovery and rehabilitation treatment plan to tackle these adverse outcomes. Where appropriate, older individuals recovering from SARS Cov2 may need to undergo a fracture risk assessment and DXA coupled to anti-osteoporosis treatment [47].

In addition, the measures implemented during the pandemic, such as the travel bans, quarantines, self-isolation has led to reduced physical activity, particularly in the elderly population predisposing to loss of muscle mass and function and contributing to sarcopenia [48].

\section{Managing Osteoporosis During Pandemic}

As reviewed above, the pandemic has presented several challenges for treating osteoporosis, particularly in patients on parenteral therapies. The concerns surround the establishment and implementation of effective alternative strategies, and the adoption of new ways of practice such as remote consultations to reduce fracture burden has been proposed [49]. There is evidence of the use of telemedicine approaches in the field of osteoporosis. A study from Canada comprising of a mailed satisfaction survey and telephone interviews to understand patient experiences of osteoporosis care delivered virtually by telemedicine showed that the patients' perception of care by telemedicine was comparable to face-to-face visits with added benefits which included convenience, reduced travel time, and costs. Nevertheless, there was a need for improvement as patients were concerned about the followup with allied professionals such as physiotherapists and 
the co-ordination of investigations and tests. They also expressed interest in the design of an osteoporosis virtual self-management program focusing on advice about diet and lifestyle factors [50]. Follow-up telephone consultations to promote adherence already forms part of the FLS in the UK, although this has been disrupted in the current pandemic. However, the effectiveness of telephone consultations on adherence rates to osteoporosis medication use has been mixed with modest effect reported in a Canadian trial and no difference seen in two American studies [51-53].

Clinical guidance on screening and treatment developed and endorsed by the American Society for Bone and Mineral Research (ASBMR), Endocrine Society, the American Association of Clinical Endocrinologists, the European Calcified Tissue Society and the European Society for Endocrinology have been published [54]. These recommendations were largely based on expert opinion as evidence-based data were lacking.

The difficulty in accessing DXA scans during the pandemic has been highlighted previously in this review. An algorithm has been suggested based on the use of FRAX in patients who had been referred for DXA. The authors suggested that this could be done through a telephone consultation during which information about the patient's age, gender, weight, height, clinical risk factors are obtained to derive the FRAX score. It was suggested that following calculation of FRAX, patients could be categorised as low risk, intermediate risk, and high risk. Subsequently, those in the intermediate-risk category can proceed with DXA examinations, those in the low-risk category can be reassured (or DXA deferred) and those in the high-risk treated without DXA [55]. This is very similar to the approach advocated in the NOGG guidance when DXA scans are unavailable or impractical. There are several potential issues with this approach, one is gaining accurate information on height, weight, and other risk factors; a second would be difficulties in communication with people who were hard of hearing and those with cognitive problems. As has been mentioned previously there is limited information on how effective treatments for osteoporosis are in patients with high fracture risk alone in the absence of information from DXA. The SCOOP study provided some insights into the effectiveness of treating individuals at high risk of fracture without DXA. During this 5-year study, $24 \%$ of individuals in the "screened" group received at least one prescription for anti-osteoporosis treatment compared with $16 \%$ of the control group. The hazard ratio for fracture was 0.94 [0.85-1.03] in the screened group a difference that was not significant. Hip fractures were less common in the screened group however $(0.72$ [0.59.0.89]) [56]. It should be noted that in SCOOP, the 10-year risk of hip fracture in both groups was about $7.5 \%$ which is considerably higher than the "treat" recommendation based on hip fracture probability in the NOGG algorithm.

The authors also suggested that the majority can be delayed based on clinical judgement, particularly if this is for monitoring patients who are on osteoporosis treatment or when previous scans have shown stable BMD and there are no new clinical risk factors [55]. It was suggested that priority should be given, however, to those who have had a significant decline in BMD on previous DXA scans or who develop new risk. While the algorithm is pragmatic the efficacy has not been tested in routine clinical care, and it is, therefore, unclear if it would reduce fracture burden in these times.

The joint guidelines of the bone health organisations addressed the management considerations, particularly those receiving treatment with iv bisphosphonates (zoledronate), denosumab, teriparatide, and romosozumab [54, 57] and are summarised in Fig. 2. There is no evidence that any of these therapeutic agents increases the risk of SARS-Cov-2 infection but a meta-analysis of 33 randomised controlled trials (22,253 patients) showed that denosumab is associated with an increased risk of severe infections, especially of ear, nose and throat infections [58]. A telephone survey of 85 patients on osteoporosis medications including denosumab attending the bone clinic in the Endocrine Division of San Raffaele Hospital Milan, one of the epicentres of COVID-19 pandemic in Italy, was undertaken between 21st February to 24th May 2020 [59]. Forty-two patients responded and 26 were on denosumab. Only 1 patient (3.8\%) on denosumab reported symptoms consistent with a respiratory tract infection during the pandemic but did not have a COVID-19 swab test. None of the patients on denosumab were hospitalised. Although, it appears that treatment with Denosumab is not a specific risk factor for COVID-19 infection and data from this small 'real-life' study support the guidelines that denosumab should continue during the pandemic, larger studies may be needed to confirm this.

There is also no evidence that any of the osteoporosis therapies interferes the efficacy or side-effect profiles of the COVID-19 vaccines. However, some minor changes to the timing of the osteoporosis drug administration may be considered to account for the patient's COVID-19 vaccine schedule [60].

\section{Maintenance and Recovery of Osteoporosis Services}

Information about the adaptations to the metabolic bone/ osteoporosis services in the authors' centres during the pandemic is provided in the supplementary material. 


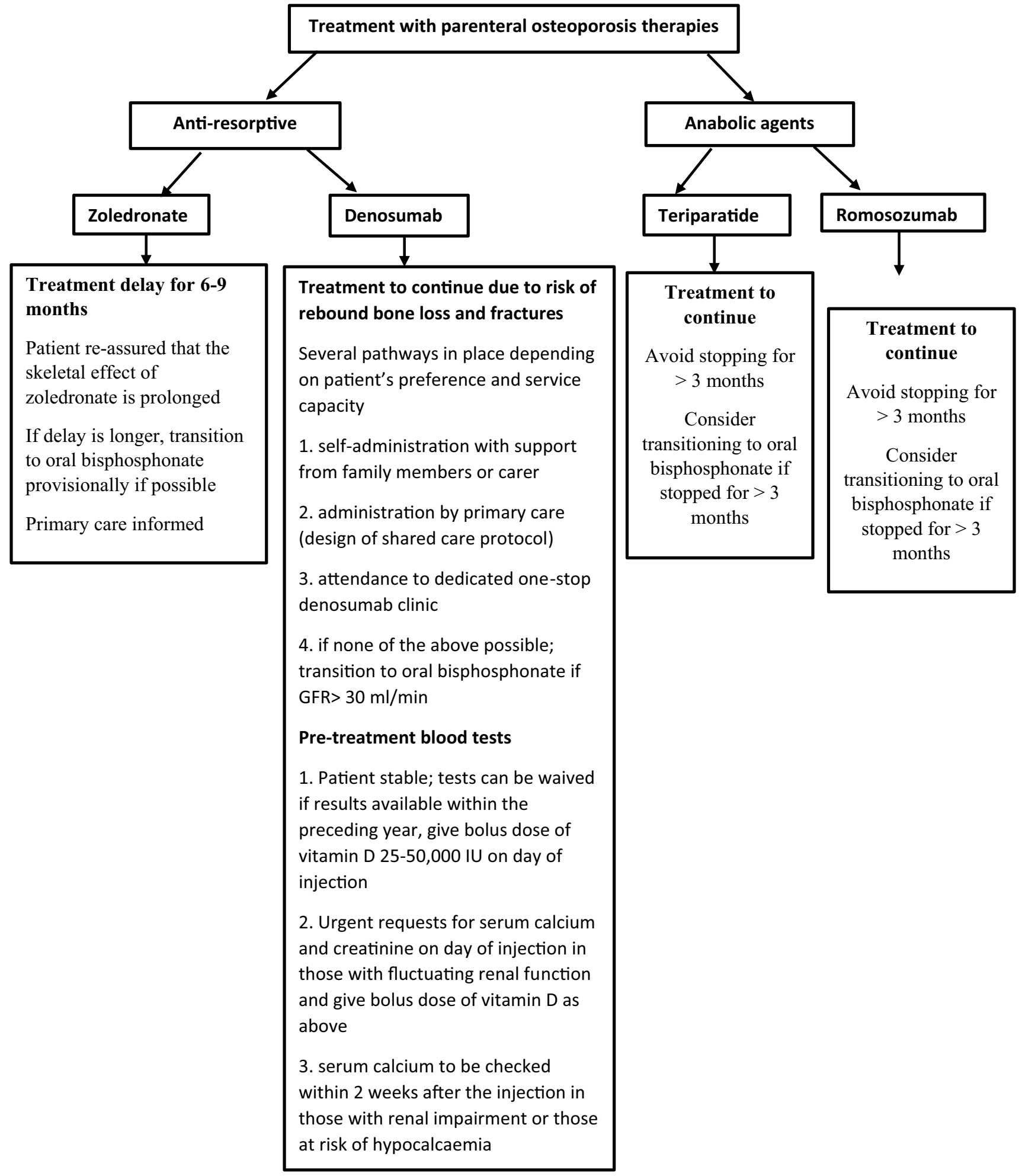

Fig. 2 Algorithm summarising the guidance to the management of osteoporosis during the COVID-19 pandemic in patients on parenteral treatment. All patients should continue calcium and/or vitamin D supplements and be encouraged to maintain a healthy-balanced diet and lifestyle such as stopping smoking, avoiding excessive alco- hol intake (>3 units/day), keeping active and exercising regularly. Adapted from Yu, E. W. et al. Osteoporosis management in the era of COVID-19 [53] and Gittoes et al. Endocrinology in the time of COVID-19: management of calcium metabolic disorders and osteoporosis [56] 


\section{Lifestyle Measures}

We continue to emphasise the importance of lifestyle measures such as maintaining a healthy balanced diet, stopping smoking and reducing alcohol intake ( $<3$ units/day) and taking regular exercise while acknowledging that this is more difficult due to restrictions on leaving home and closing of facilities like leisure centres and gyms. To mitigate this, home-based resistance exercise interventions aimed at increasing physical activity could be implemented through telehealth and interaction with a physiotherapist and could also include the use of educational exercise videos [48].

\section{Vitamin D Supplements}

Because of the fact that frail older individuals may be shielding and unable to get outside we endorse the advice for vitamin $\mathrm{D}$ supplementation and calcium where required if dietary intake is low. For patients receiving intravenous bisphosphonates higher dose supplements can be used such as a bolus doses of cholecalciferol (25-50,000 IU) a few days before treatment since these have been shown to increase serum $25(\mathrm{OH})$ vitamin D concentrations within three days after oral supplementation [61]. Doses, however, probably should not exceed 50,000 IU as bolus doses of 500,000 IU annually and 60, $000 \mathrm{IU}$ monthly were associated with increased risk of falls and fractures in populations who were not vitamin D deficient [62, 63].

Observational studies report an association between vitamin D deficiency and susceptibility to respiratory infections due to potential benefits on the immune system; data in the context of COVID-19 are inconsistent and evidence for causality is lacking [64, 65]. There are several ongoing trials investigating the effect of vitamin D supplementation on COVID-19 prevention and management which may help provide further guidance [64].

\section{Intravenous Bisphosphonates}

Due to the long half-life of bisphosphonates in the bone we advise that delaying infusions for time periods of up 12 months is unlikely to lead to increased fracture risk or significant decline in BMD as zoledronate is long-acting [30-33]. While transitioning to oral bisphosphonates is theoretically possible, our collective experience is that in most patients, intravenous therapy has been started because of intolerance or contraindications to oral bisphosphonates. As is normal practice it is important to inform patients of the acute phase response (APR) with intravenous bisphosphonates but perhaps even more so as the symptoms can be mistaken for those of SARS-Cov2 infection. As a pragmatic workaround, we advise that patients should not consider being tested for SARS-Cov2 after intravenous bisphosphonate unless the symptoms persist for more than 4 days.

\section{Denosumab}

We strongly advise that denosumab treatment delay should not exceed seven months since the last dose $[34,35]$. We also urge caution regarding commencement of denosumab unless other options are unsuitable. Where possible a shared care service should be agreed or one-stop clinics established in suitable areas of the hospital with appropriate social distancing measures in place.

\section{Anabolic Drugs}

For patients who are on the anabolic agents teriparatide or romosozumab, treatment should be continued as these are self-administered injections. If, however, treatment has to be discontinued or patients are coming to the end of their course of treatment it is advised that they transition to oral bisphosphonate in the first instance.

\section{Biochemical Investigations}

It may be possible to introduce longer intervals between routine blood tests and parenteral treatments such as intravenous bisphosphonates unless there is concern about fluctuating renal function. One option is to have a window of 3-4 months in low-risk patients with eGFR $>40$ but to require testing before treatment in a shorter interval of $7-10$ days in those with eGFR $<40$. Checks of $25(\mathrm{OH}) \mathrm{D}$ may not be required in patients who are taking vitamin $\mathrm{D}$.

\section{Initiating Treatment in the Absence of DXA}

It is possible to advise treatment in the absence of DXA. If this is done there is some evidence that in elderly women with a 10-year fracture risk of $>20 \%$ who are prescribed oral bisphosphonates have a reduction in hip fracture risk but not the risk of other fractures [54]. If a decision is made to start treatment DXA should be performed when feasible, fracture risk recalibrated and the need for treatment reviewed. In patients with a recent hip fracture, there is good evidence than zoledronic acid reduces the risk of further fractures [66]. Such evidence is lacking for other treatments.

\section{Managing the Hip Fracture Patient}

Quality improvement programs can be put in place to increase awareness of bone health assessment among junior doctors and nurse practitioners. This approach has been shown to improve the management of patients with femoral neck fractures as the prescription rates for calcium/vitamin 
$\mathrm{D}$, bone-sparing drugs and DXA scan requests increased following the implementation of the tool [27]. Access to rehabilitation should be made available with application of the required social distancing and infection control measures. In cases of patients admitted with a hip fracture who are also affected with COVID-19 where mortality has been shown to be high as previously described [26, 44], orthopaedic departments should organise specific accelerated care pathways for their treatment to reduce length of stay in hospital or requirement for intensive care bed [67, 68]. In a recent study of 16 patients admitted with femoral neck fractures and COVID-19 infection, orthopaedic surgery on the day of or within three days of admission contributed to patients' haemodynamic and respiratory stability, improvement in respiratory function and comfort in bed [69].

Bone health review should also include the assessment of spinal deformity, severity and acuteness of back pain, review of chest X-rays and/or CT scans for the opportunistic identification of prevalent vertebral fractures (VFs) with appropriate management offered including pain relief, spinal support [70].

\section{Ensuring Patients are not Lost to Follow Up}

Recall procedures must be put in place to make sure that patients do not get missed for the administration of osteoporosis drugs and clinical assessment when routine services resume safely in the aftermath of the pandemic when patients have had access to vaccination.

\section{Conclusions}

The screening, diagnosis and management of patients with osteoporosis have proved to be challenging during the COVID-19 pandemic. The follow-up of patients to the metabolic bone clinics has been disrupted which may create future problems due to treatment delays, particularly in patients on denosumab. The quality of osteoporosis care of patients with a new fragility fracture or hip fracture following surgery has dramatically decreased during the pandemic [71].

To avoid the negative impact that the disruption to healthcare services can have on future fracture burden, new guidance about osteoporosis treatment has been issued which involves the design of new care pathways. The new recommendations offer a pragmatic approach to the delivery of osteoporosis services which aim to ensure that the best level of care for fracture prevention is maintained during these unprecedented times. As effective vaccination gets underway and the pandemic is controlled, the alternative models of care instituted such as remote consultations, telehealth medicine, better co-ordination of primary and secondary care, and sharing of resources, should help and incentivise us in building more robust, patient-friendly systems of service delivery.

Supplementary Information The online version contains supplementary material available at https://doi.org/10.1007/s00223-021-00858-9.

Author Contributions GH thought of the idea and drafted the manuscript, SHR further developed the idea and revised the first draft, JRL, MS, RKC further revised the manuscript and wrote their respective centre's experience. All authors reviewed the manuscript for content and approved the final version.

\section{Declarations}

Conflict of interest GH: speakers fee Amgen, advisory board fee UCB and Kyowa-Kirin International Ltd. JRL: speakers fee Amgen and Consilient Healthcare, advisory board fee UCB. MDS: speaker and consultancy fees Amgen, Gedeon Richter, UCB; member of NOGG expert advisory group; member of Royal Osteoporosis Society Clinical Committee. RKC: advisory board UCB; investigator Kiowa Kirin. SHR: research.

Open Access This article is licensed under a Creative Commons Attribution 4.0 International License, which permits use, sharing, adaptation, distribution and reproduction in any medium or format, as long as you give appropriate credit to the original author(s) and the source, provide a link to the Creative Commons licence, and indicate if changes were made. The images or other third party material in this article are included in the article's Creative Commons licence, unless indicated otherwise in a credit line to the material. If material is not included in the article's Creative Commons licence and your intended use is not permitted by statutory regulation or exceeds the permitted use, you will need to obtain permission directly from the copyright holder. To view a copy of this licence, visit http://creativecommons.org/licenses/by/4.0/.

\section{References}

1. Coronavirus disease (COVID-19) Weekly epidemiological update and weekly operational update.https://www.who.int/emergencies/ diseases/novel-coronavirus-2019/situation-reports.

2. Thevarajan I, Nguyen THO, Koutsakos M, Druce J, Caly L, van de Sandt CE, Jia X, Nicholson S, Catton M, Cowie B et al (2020) Breadth of concomitant immune responses prior to patient recovery: a case report of non-severe COVID-19. Nat Med 26:453-455

3. Wu Z, McGoogan JM (2020) Characteristics of and important lessons from the coronavirus disease 2019 (COVID-19) outbreak in China: summary of a report of 72,314 cases from the Chinese center for disease control and prevention. JAMA 323:1239-1242

4. Huang C, Wang Y, Li X, Ren L, Zhao J, Hu Y et al (2020) Clinical features of patients infected with 2019 novel coronavirus in Wuhan, China. Lancet 395:497-506

5. Rosenbaum L (2020) The untold toll-the pandemic's effect on patients without COVID-19. N Engl J Med. https://doi.org/10. 1056/NEJMms2009984

6. Center for Disease Control and Prevention (2020) Framework for healthcare systems providing non-COVID-19 clinical care during the COVID-19 pandemic. 2019-ncov/hcp/framework- nonCOVID- care.html (CDC, 2020). https://www.cdc.gov/coron avirus/. Accessed 10 Jan 2021 
7. Hernlund E, Svedbom A, Ivergard M, Compston J, Cooper C, Stenmark J et al (2013) Osteoporosis in the European union: medical management, epidemiology and economic burden. A report prepared in collaboration with the international osteoporosis foundation (IOF) and the European federation of pharmaceutical industry associations (EFPIA). Arch Osteoporos 8:136

8. Conley RB, Adib G, Adler RA, Akesson KE, Alexander IM, Amenta KC et al (2020) Secondary fracture prevention: consensus clinical recommendations from a multistakeholder coalition. J Bone Min Res 35(1):36-52

9. Compston J, Cooper A, Cooper C, Gittoes N, Gregson C, Harvey $\mathrm{N}$ et al (2017) UK clinical guideline for the prevention and treatment of osteoporosis. Arch Osteoporos 12(1):43

10. Scottish Intercollegiate Guideline Network (2015) Management of osteoporosis and the prevention of fragility fractures. Edinburgh: SIGN. (SIGN Guideline No. 142). Available at www. sign.ac.uk/our-guidelines/management-of-osteoporosis-and-theprevention-of-fragility-fractures. Accessed 12 Feb 2021

11. Hippisley-Cox J, Coupland C (2012) Derivation and validation of updated QFracture algorithm to predict risk of osteoporotic fracture in primary care in the United Kingdom: prospective open cohort study. BMJ 344:e3427

12. Nguyen ND, Frost SA, Center JR, Eisman JA, Nguyen TV (2008) Development of prognostic nomograms for individualizing 5-year and 10-year fracture risks. Osteoporos Int 19(10):1431-1444

13. Kanis JA On behalf of the World Health Organization Scientific Group (2008) Assessment of osteoporosis at the primary healthcare level. Technical report. (https://www.sheffield.ac.uk/FRAX/ pdfs/WHO_Technical_Report.pdf). University of Sheffield, UK

14. Kanis JA, Harvey NC, Cooper C, Johansson H, Oden A, McCloskey EV, Advisory Board of the National Osteoporosis Guideline G (2016) A systematic review of intervention thresholds based on FRAX: a report prepared for the national osteoporosis guideline group and the international osteoporosis foundation. Arch Osteoporos 11:25

15. Chotiyarnwong $\mathrm{P}$, Harvey NC, Johansson H, Liu E, Lorentzen M, Kanis JA, McCloskey EV (2019) Temporal changes in access to FRAX(R) in Thailand between 2010 and 2018. Arch Osteoporos $14: 66$

16. McCloskey EV, Harvey NC, Johansson H, Lorentzon M, Vandenput L, Liu E et al (2021) Global impact of COVID-19 on noncommunicable disease management: descriptive analysis of access to FRAX fracture risk online tool for prevention of osteoporotic fractures. Osteoporos Int 32(1):39-46

17. Camacho PM, Petak SM, Binkley N, Diab DL, Eldeiry LS, Farooki A et al (2020) American association of clinical endocrinologists/American college of endocrinology clinical practice guidelines for the diagnosis and treatment of postmenopausal osteoporosis- 2020 update executive summary. Endocr Pract 26(5):564-570

18. National Health Service (NHS), England. Monthly diagnostic waiting times. Available at https://www.england.nhs.uk/stati stics/statistical-work-areas/diagnostics-waiting-times-and-activ ity/monthly-diagnostics-waiting-times-and-activity/monthlydia gnostics-data-2020-21/.

19. McLellan AR, Gallacher SJ, Fraser M, McQuillian C (2003) The fracture liaison service: success of a program for the evaluation and management of patients with osteoporotic fracture. Osteoporos Int 14(12): 1028-1034

20. Geusens P, Bours SPG, Wyers CE, Van den Bergh JP (2019) Fracture liaison programs. Best Pract Res Clin Rheumatol 33:278-289

21. Axelsson KF, Johansson H, Lundh D, Möller M, Lorentzon M (2020) Association between recurrent fracture risk and implementation of fracture liaison services in four Swedish hospitals: a cohort study. J Bone Miner Res 35:1216-1223
22. Ogliari G, Lunt E, Ong T, Marshall L, Sahota O (2020) The impact of lockdown during the COVID-19 pandemic on osteoporotic fragility fractures: an observational study. Arch Osteoporos 15(1): 156

23. English S, Coyle L, Bradley S, Wilton W, Cordner J, Dempster $R$ et al (2021) Virtual fracture liaison clinics in the COVID era: an initiative to maintain fracture prevention services during the pandemic associated with positive patient experience. Osteoporos Int. https://doi.org/10.1007/s00198-021-05882-x

24. Maniscalco P, Poggiali E, Quattrini F et al (2020) Proximal femur fractures in COVID-19 emergency: the experience of two orthopedics and traumatology departments in the first eight weeks of the Italian epidemic. Acta Biomed 91(2):89-96

25. Malik-Tabassum K, Crooks M, Robertson A, To C, Maling L, Selmon G (2020) Management of hip fractures during the COVID-19 pandemic at a high-volume hip fracture unit in the United Kingdom. J Orthop 20:332-337

26. Arafa M, Nesar S, Abu-Jabeh H, Jayme MOR, Kalairajah Y (2020) COVID-19 pandemic and hip fractures: impact and lessons learned. Bone Jt Open 1(9):530-540

27. Stephens A, Rudd H, Stephens E, Ward J (2020) Secondary prevention of hip fragility fractures during the COVID-19 pandemic: service evaluation of "MRS BAD BONES." JMIR Aging 3(2): 25607

28. Black DM et al (2018) The ability of a single BMD and fracture history assessment to predict fracture over 25 years in postmenopausal women: the study of osteoporotic fractures. J Bone Miner Res 33:389-395

29. Black DM, Reid IR, Boonen S et al (2012) The effect of 3 versus 6 years of zoledronic acid treatment of osteoporosis: a randomized extension to the HORIZON-pivotal fracture trial (PFT). J Bone Miner Res 27(2):243-254

30. Grey A, Bolland MJ, Horne A (2012) Five years of antiresorptive activity after a single dose of zoledronate-results from a randomized doubleblind placebo-controlled trial. Bone 50(6):1389-1393

31. Grey A, Horne A, Gamble G, Mihov B, Reid IR, Bolland AM (2020) Ten years of very infrequent zoledronate therapy in older women: an open-label extension of a randomized trial. J Clin Endocrinol Metab 105(4):e1641-e1647

32. Watts NB, Chines A, Olszynski WP (2008) Fracture risk remains reduced one year after discontinuation of risedronate. Osteoporos Int 19(3):365-372

33. Schwartz AV, Bauer DC, Cummings SR et al (2010) Efficacy of continued alendronate for fractures in women with and without prevalent vertebral fracture: the FLEX trial. J Bone Miner Res 25(5):976-982

34. Bone HG, Bolognese MA, Yuen CK et al (2011) Effects of denosumab treatment and discontinuation on bone mineral density and bone turnover markers in postmenopausal women with low bone mass. J Clin Endocrinol Metab 96(4):972-980

35. Cummings SR, Ferrari S, Eastell R et al (2018) Vertebral fractures after discontinuation of denosumab: a post hoc analysis of the randomized placebo-controlled FREEDOM trial and its extension. J Bone Miner Res 33(2):190-198

36. Tsourdi E, Langdahl B, Cohen-Solal M, Aubry-Rozier B, Eriksen EF, Guanabens N et al (2017) Discontinuation of Denosumab therapy for osteoporosis: a systematic review and position statement by ECTS. Bone 105:11-17

37. Leder BZ, Neer RM, Wyland JJ, Lee HW, Burnett-Bowie SM, Finkelstein JS (2009) Effects of teriparatide treatment and discontinuation in postmenopausal women and eugonadal men with osteoporosis. J Clin Endocrinol Metab 94(8):2915-2921

38. Cosman F, Nieves JW, Zion M et al (2015) Daily or cyclical teriparatide treatment in women with osteoporosis on no prior 
therapy and women on alendronate. J Clin Endocrinol Metab 100(7):2769-2776

39. Oswald AJ, Berg K, Ralston SH, Riches PL (2019) Long-term effects of teriparatide followed by antiresorptive therapy on clinical outcomes in patients with severe spinal osteoporosis. Calcif Tissue Int 105(2):148-155

40. McClung MR, Brown JP, Diez-Perez A et al (2018) Effects of 24 months of treatment with romosozumab followed by 12 months of denosumab or placebo in postmenopausal women with low bone mineral density: a randomized, double-blind, phase 2, parallel groups study. J Bone Miner Res 33(8):1397-1406

41. Williamson EJ, Walker AJ, Bhaskaran K, Bacon S, Bates C, Morton CE et al (2020) Factors associated with COVID-19-related death using OpenSAFELY. Nature 584(7821):430-436

42. Clift AK, Coupland CAC, Keogh RH, Diaz-Ordaz K, Williamson E, Harrison EM et al (2020) Living risk prediction algorithm (QCOVID) for risk of hospital admission and mortality from coronavirus 19 in adults: national derivation and validation cohort study. BMJ 371:m3731

43. di Filippo L, Formenti AM, Doga M, Pedone E, Rovere-Querini P, Giustina A (2020) Radiological thoracic vertebral fractures are highly prevalent in COVID-19 and predict disease outcomes. J Clin Endocrinol Metab. https://doi.org/10.1210/clinem/dgaa738

44. Genant HK, Jergas M, Palermo L, Nevitt M et al (1996) Comparison of semiquantitative visual and quantitative morphometric assessment of prevalent and incident vertebral fractures in osteoporosis. The study of osteoporotic fractures research group. Bone Miner Res 11(7):984-996

45. Clement ND, Ng N, Simpson CJ, Patton RFL, Hall AJ, Simpson A et al (2020) The prevalence, mortality, and associated risk factors for developing COVID-19 in hip fracture patients: a systematic review and meta-analysis. Bone Joint Res 9(12):873-883

46. After-care-needs-of-inpatients-recovering-from-COVID19-5-june-2020-1.pdf https://www.england.nhs.uk/coronavirus/ wp-content/uploads/sites/52/2020/06/C0388.

47. Napoli N, Elderkin AL, Kiel DP, Khosla S (2020) Managing fragility fractures during the COVID-19 pandemic. Nat Rev Endocrinol 16(9):467-468

48. Kirwan R, Mc Cullough D, Butler T, Perez de Heredia F, Davies IG, Stewart C (2020) Sarcopenia during COVID-19 lockdown restrictions: long-term health effects of short-term muscle loss. GeroScience 42:1547-1578

49. Paskins Z, Crawford-Manning F, Bullock L, Jinks C (2020) Identifying and managing osteoporosis before and after COVID-19: rise of the remote consultation? Osteoporos Int 31(9):1629-1632

50. Palcu P, Munce S, Jaglal SB, Allin S, Chishtie JA, Silverstein A, Kim S (2020) Understanding patient experiences and challenges to osteoporosis care delivered virtually by telemedicine: a mixed methods study. Osteoporos Int 31:351-361

51. Jaglal SB, Donescu OS, Bansod V, Laprade J, Thorpe K, Hawker G, Majumdar SR, Meadows L, Cadarette SM, Papaioannou A, Kloseck M, Beaton D, Bogoch E, Zwarenstein M (2012) Impact of a centralized osteoporosis coordinator on post-fracture osteoporosis management: a cluster randomized trial. Osteoporos Int 23:87-95

52. Schousboe J, DeBold R, Kuno L, Weiss T, Chen Y, Abbott T (2005) Education and phone follow-up in postmenopausal women at risk for osteoporosis: effects on calcium intake, exercise frequency, and medication use. Dis Manag Health Outcomes 13:395-404

53. Solomon DH, Iversen MD, Avorn J, Gleeson T, Brookhart MA, Patrick AR, Rekedal L, Shrank WH, Lii J, Losina E, Katz JN (2012) Osteoporosis telephonic intervention to improve medication regimen adherence: a large, pragmatic, randomized controlled trial. Arch Intern Med 172:477-483
54. Yu EW, Tsourdi E, Clarke BL, Bauer DC, Drake MT (2020) Osteoporosis management in the era of COVID-19. J Bone Miner Res. https://doi.org/10.1002/jbmr.4049

55. Sapkota HR, Nune A, Bateman J, Venkatachalam S (2021) A pragmatic proposal for triaging DXA testing during the COVID19 global pandemic. Osteoporos Int 32(1):1-6

56. Shepstone L, Lenaghan E, Cooper C, Clarke S, Fong-Soe-Khioe $\mathrm{R}$, Fordham R et al (2018) Screening in the community to reduce fractures in older women (SCOOP): a randomised controlled trial. Lancet 391(10122):741-747

57. Gittoes NJ, Criseno S, Appelman-Dijkstra NM, Bollerslev J, Canalis E, Rejnmark L et al (2020) Endocrinology in the time of COVID-19: management of calcium metabolic disorders and osteoporosis. Eur J Endocrinol 183(2):G57-G65

58. Diker-Cohen T, Rosenberg D, Avni T, Shepshelovich D, Tsvetov G, Gafter-Gvili A (2020) Risk for infections during treatment with denosumab for osteoporosis: a systematic review and metaanalysis. J Clin Endocrinol Metab 105(5):1641-1658

59. Formenti AM, Pedone E, di Filippo L, Ulivieri FM, Giustina A (2020) Are women with osteoporosis treated with denosumab at risk of severe COVID-19? Endocrine 70(2):203-205

60. Joint Guidance on COVID-19 Vaccination and Osteoporosis Management from the American Society for Bone and Mineral Research (ASBMR), American Association of Clinical Endocrinology (AACE), Endocrine Society, European Calcified Tissue Society (ECTS), the International Osteoporosis Foundation (IOF), and the National Osteoporosis Foundation (NOF). https://www. asbmr.org/about/statement-detail/joint-guidance-on-COVID-19vaccine-osteoporosis.

61. Romagnoli E, Mascia ML, Cipriani C, Fassino V, Mazzei F, D’Erasmo E, Carnevale V, Scillitani A, Minisola S (2008) Short and long-term variations in serum calciotropic hormones after a single very large dose of ergocalciferol (vitamin D2) or cholecalciferol (D3) in the elderly. J Clin Endocrinol Metab 93:3015-3020

62. Sanders KM, Stuart AL, Williamson EJ, Simpson JA, Kotowicz MA, Young D, Nicholson GC (2010) Annual high-dose oral vitamin $\mathrm{D}$ and falls and fractures in older women: a randomized controlled trial. JAMA 303:1815-1822

63. Bischoff-Ferrari HA, Dawson-Hughes B, Orav EJ, Staehelin HB, Meyer OW, Theiler R, Dick W, Willett WC, Egli A (2016) Monthly high-dose vitamin $d$ treatment for the prevention of functional decline: a randomized clinical trial. JAMA Intern Med 176:175-183

64. Vimaleswaran KS, Forouhi NG, Khunti K (2021) Vitamin D and COVID-19. BMJ 372:n544

65. Tramontana F, Napoli N, El-Hajj Fuleihan G, Strollo R (2020) The D-side of COVID-19: musculoskeletal benefits of vitamin D and beyond. Endocrine 69(2):237-240

66. Lyles KW, Colon-Emeric CS, Magaziner JS, Adachi JD, Pieper CF, Mautalen C et al (2007) Zoledronic acid and clinical fractures and mortality after hip fracture. N Engl J Med 357(18):1799-1809

67. Tarantino U, Cariati I, Tancredi V, Casamassima D, Piccirilli E, Iundusi R et al (2020) State of fragility fractures management during the COVID-19 pandemic. Int J Environ Res Public Health 17(21):7732

68. Kumar Jain V, Lal H, Kumar Patralekh M, Vaishya R (2020) Fracture management during COVID-19 pandemic: a systematic review. J Clin Orthop Trauma 11(Suppl 4):S431-S441

69. Catellani F, Coscione A, D’Ambrosi R, Usai L, Roscitano C, Fiorentino G (2020) Treatment of proximal femoral fragility fractures in patients with COVID-19 during the SARS-CoV-2 outbreak in Northern Italy. J Bone Joint Surg Am 102:e58

70. Clinical guidance for the effective identification of vertebral fractures. https://www.guidelines.co.uk/musculoskeletal-and-joints-/ ros-guideline-identification-of-vertebral-fractures/454148.article. 
71. Peeters JJM, van den Berg P, van den Bergh JP, Emmelot-Vonk MH, de Klerk G, Lems WF et al (2021) Osteoporosis care during the COVID-19 pandemic in the Netherlands: a national survey. Arch Osteoporos 16(1):11
Publisher's Note Springer Nature remains neutral with regard to jurisdictional claims in published maps and institutional affiliations. 